Notre Dame Journal of Formal Logic

Volume XIII, Number 2, April 1972

NDJFAM

\title{
THE PRAGMATICS OF FIRST ORDER LANGUAGES. I
}

\section{ALBERT SWEET}

1. Introduction: Pragmatic Interpretations According to the well-known semiotic principle of Peirce, every signification is a triadic relation between sign, object, and interpretant. In this paper we shall investigate the structure of this relation, in the case where the signs are formulas of a first order language, their objects are taken to be elements in a model of the language, and their interpretants are taken to be certain valuing dispositions of the users of the language. We shall show that there are pragmatically definable linguistic models of the theory of polyadic Boolean algebras [1]. Then we may represent first order languages by such linguistic algebras, and their models by appropriate functional algebras; we shall represent the relevant valuing dispositions of the users of first order languages by means of pragmatic interpretations, which we now define.

Let $L$ be the set of all finite sequences of the elements of a given non-empty finite set $L_{0}$. Let $V$ be a three-membered set, which we represent as $\{0,1,2\}$. Let $C, U, W$ be arbitrary non-empty sets, disjoint from one another and from $L$ and $V$. Intuitively, $L$ is the set of expressions of a language with alphabet $L_{0}, C$ is a set of conditions under which the expressions of $L$ may be valued in the set $\mathbf{V}$ by the members of the set $U$ of users of $L$, and $W$ is the set of times at which expressions of $L$ may be valued. The elements $0,1,2$ of $\mathrm{V}$ may be regarded as, respectively, the values reject, accept, neither accept nor reject. The set $\mathrm{C}$ may be regarded as the total evidence available to the users of $L$, and subsets of $C$ as partial evidence, for valuing the expressions of $L$ in the set $V$.

Let $\mathbb{S}$ be the set of all subsets of $C$. Let $D$ be the set of all mappings $d$ from the Cartesian product $\mathbf{U} \times \mathbf{W} \times \mathfrak{C}$ into $\mathbf{V}$ such that, for all $u, u^{\prime} \in \mathbf{U}$; $w, w^{\prime} \in \mathbf{W} ; c \subset \mathbf{C}$ :

(1.1) $d(u, w, c)=d\left(u^{\prime}, w^{\prime}, c\right)$ if $d(u, w, c) \neq 2 \neq d\left(u^{\prime}, w^{\prime}, c\right)$.

An element $(u, w, c, v)$ of a mapping $d$ in $D$ may be regarded as a disposition of the user $u$, at time $w$, under the set of conditions $c$, to perform the valuation $v$. (1.1) is a condition of uniformity on the mappings $d$ in $D$, in the 
sense that such $d$ will contain no dispositions to perform distinct valuations under conditions germane for the valuations, i.e., under conditions with respect to which accepting or rejecting valuations are performed. The constant function in $d$ which maps all $(u, w, c)$ on 0 , we shall call $d_{0}$; similarly for $d_{1}$ and $d_{2}$.

It is natural to regard appropriate functions $\Pi$ from $\mathbf{L}$ into $D$ as pragmatic interpretations of $L$, insofar as $\Pi$ assigns to each expression of $\mathrm{L}$ a set of valuing dispositions. If $\Pi$ is a mapping from $\mathrm{L}$ into $D$ such that there are expressions $e, e^{\prime}, e^{\prime \prime}$ in $\mathrm{L}$ for which

$$
\Pi(e)=d_{0} ; \Pi\left(e^{\prime}\right)=d_{1} ; \Pi\left(e^{\prime \prime}\right)=d_{2}
$$

then we shall say that $\Pi$ is a pragmatic interpretation of $L$. (1.2) insures the existence of what we shall call the "core" of a pragmatic interpretation: if $\Pi$ is a pragmatic interpretation of $L$, we define the core of $\Pi$ to be the mapping $\pi$ from $L \times \mathbb{E}$ onto $V$ such that:

$$
\text { (1.3) } \pi(e, c)=\left\{\begin{array}{l}
2, \text { if } \Pi(e)(u, w, c)=2 \text { for all } u \in \mathbf{U}, w \in \mathbf{W} . \\
0(1) \text {, if } \Pi(e)(u, w, c)=0(1) \text { for some } u \in \mathbf{U}, w \in \mathbf{W} .
\end{array}\right.
$$

for all $e \epsilon \mathrm{L}, c \subset C$, where $\Pi(e)(u, w, c)$ is the value of the function $\Pi(e)$ for the argument $(u, w, c)$ in $\mathbf{U} \times \mathbf{W} \times \mathfrak{c}$. The single-valued property of $\pi$ defined by (1.3) follows from (1.1). From (1.3) we also have, for all $e \epsilon \mathrm{L}, c \subset \mathbb{}$ :

$$
\text { (1.4) If } \Pi(e)=\Pi\left(e^{\prime}\right) \text {, then } \pi(e, c)=\pi\left(e^{\prime}, c\right) \text {. }
$$

We shall often employ (1.4) without explicit mention of the fact.

The core of a pragmatic interpretation $\Pi$ of $L$ represents the structure of $\Pi$ which is invariant under transformations on $\Pi$, regarded as a set of ordered quintuples $(e, u, w, c, v)$, which leave $e$ and $c$ unchanged: the values $v \neq 2$ are preserved under such transformations. It is this invariant property of pragmatic interpretations which, we assume, may determine objects of the signs of $L$ which are uniformly grasped by their users. We proceed now to clarify this idea in the following manner.

A pragmatic interpretation of $L$ which induces on an appropriate subset of $L$ the structure of a polyadic Boolean algebra, we shall call a polyadic interpretation of L. Polyadic interpretations are generalizations of sentential interpretations, which are studied in section 2. Polyadic interpretations are studied in section 3. In section 4 it is shown that a polyadic interpretation of $L$ induces on an appropriate subset of $L$ the structure of a polyadic Boolean algebra. Finally in section 5 the results of the preceding sections are applied to the problem of characterizing the structure of the sign relation.

2. Sentential Interpretations Pragmatic interpretations of $L$ which induce on an appropriate subset of $L$ the structure of a Boolean algebra, we shall call sentential interpretations. It is convenient first to define the concept of a proto-sentential interpretation, the motivation for which will become evident subsequently. We shall refer to a sequence of expressions in $L$ by juxtaposing the terms which refer to the elements of the sequence. 
D1. II is a proto-sentential interpretation of $\mathbf{L}$ iff $\Pi$ is a pragmatic interpretation of $\mathrm{L}$ and there are distinct elements \& and $\sim$ of $\mathrm{L}$ such that for all $s, s^{\prime}$ in $\mathrm{L}$ :

I. If $\Pi(s)(u, w, c) \neq 2$ for some $u \in \mathbf{U}, w \in \mathbf{W}, c \subset \mathbf{C}$, then $\Pi(\& s \sim s)=d_{0}$.

II. If $s$ contains only \& and $\sim$, then $\Pi(s)=d_{2}$.

III. If $\Pi(\& s \sim s)=d_{0}=\Pi\left(\& s^{\prime} \sim s^{\prime}\right)$, then $\Pi(\& \sim s \sim \sim s)=d_{0}=\Pi\left(\& \& s s^{\prime} \sim \& s s^{\prime}\right)$. IV. If $\Pi(\& s \sim s)=d_{0}=\Pi\left(\& s^{\prime} \sim s^{\prime}\right)$, then for all $(u, w, c)$ in $\mathbf{U} \times \mathbf{W} \times \mathfrak{c}$, the values of $\Pi(\sim s)$ and $\Pi\left(\& s s^{\prime}\right)$ and (partially) fixed by the values of $\Pi(s)$ and $\Pi\left(s^{\prime}\right)$ according to the tables:

\begin{tabular}{|c|c|c|c|c|c|}
\hline & $\sim$ & $\&$ & 0 & 1 & 2 \\
\hline 0 & 1 & 0 & 0 & 0 & 0 \\
\hline 1 & 0 & 1 & 0 & 1 & 2 \\
\hline 2 & 2 & 2 & 0 & 2 & $(\neq 1)$ \\
\hline
\end{tabular}

V. For all $c \subset \mathbf{C}$, if $\Pi(s)(u, w, c)=1$ for some $u \in \mathbf{U}, w \in \mathbf{W}$ and $\Pi\left(s^{\prime}\right)(u, w, c)=$ 1 for some $u \in \mathbf{U}, w \in \mathbf{W}$, then $\Pi\left(\& s s^{\prime}\right)(u, w, c)=1$ for some $u \in \mathbf{U}, w \in \mathbf{W}$.

If $\Pi$ is a proto-sentential interpretation of $L$, we define:

(2.1) $\mathrm{S}=\left\{s \in \mathrm{L}: \Pi(\& s \sim s)=d_{0}\right\}$.

By D1 (II): \&, $\not \mathbf{S}$. By D1 (III): if $s, s \in \mathbf{S}$, then so are $\sim s$ and $\& s s^{\prime}$. By D1 (I) and (1.2), $\mathbf{S}$ is non-empty. If $s, s^{\prime} \in \mathbf{S}$ and $\Pi(s)(u, w, c)=2=$ $\Pi\left(s^{\prime}\right)(u, w, c)$, then $\Pi\left(\& s s^{\prime}\right)(u, w, c)$ is not uniquely determined by the table for \&: at the argument $(u, w, c), \Pi\left(\& s s^{\prime}\right)$ may be either 0 or 2 . This ambiguity may be diminished by further conditions on $\Pi$, as in the subsequent definition of sentential interpretations; but functionality of the table for \& is at any rate not required for the results at which we are aiming. It is rather the functionality of pragmatic interpretations (and their cores) which will prove fruitful.

We proceed now to the definition of sentential interpretations. For perspicuity in referring to expressions of $\mathbf{S}$, we introduce the following definitions: " $(s) \&\left(s^{\prime}\right)$ " for "\&ss", and " $(s)$ ", for " $\sim s$ ", where $s, s^{\prime} \in \mathbf{S}$. We shall omit brackets introduced by such definitions when this convenience is unambiguous. It is also useful to introduce the following substitutivity notation. Let $e\left(e^{\prime}\right)$ be an expression of $\mathrm{L}$ containing any number of occurrences of $e^{\prime}$. Then for all $e^{\prime \prime}$ in $\mathrm{L}$ we define:

$$
\left\{\begin{array}{l}
e\left(e^{\prime \prime}\right) \text { expresses ambiguously any result of substituting } \\
e^{\prime \prime} \text { for } e^{\prime} \text { at any number of occurrences in } e\left(e^{\prime}\right) .
\end{array}\right.
$$

In the following definition $S$ is to be understood in the sense of (2.1).

D2. $\Pi$ is a sentential interpretation of $L$ iff $\Pi$ is a proto-sentential interpretation of $\mathrm{L}$ and for all $e \in \mathrm{L} ; s, s^{\prime}, s^{\prime \prime} \in \mathbf{S} ; c \subset \mathbf{C} ; u \in \mathbf{U} ; w \in \mathbf{W}$ :

I. If $\Pi\left(s \& \sim s^{\prime}\right)(u, w, c)=0=\Pi\left(s^{\prime} \& \sim s^{\prime \prime}\right)(u, w, c)$, then $\Pi\left(s \& \sim s^{\prime \prime}\right)(u, w, c)=0$.

II. If $\Pi\left(s \& \sim s^{\prime}\right)(u, w, c)=0$, then $\Pi\left(\left(s \& s^{\prime \prime}\right) \& \sim\left(s^{\prime} \& s^{\prime \prime}\right)\right)(u, w, c)=0$. 
III. $\Pi(e(s))=\Pi(e(\sim \sim s))$.

IV. $\Pi\left(e\left(s \& s^{\prime}\right)\right)=\Pi\left(e\left(s^{\prime} \& s\right)\right)$.

V. $\Pi\left(e\left(\left(s \& s^{\prime}\right) \& s^{\prime \prime}\right)\right)=\Pi\left(e\left(s \&\left(s^{\prime} \& s^{\prime \prime}\right)\right)\right)$.

VI. $\Pi(e(s))=\Pi(e(s \& s))$.

Let $\pi$ be the core of a sentential interpretation of $L$. Then $\pi$ determines a natural equivalence relation $\mathbf{R}_{\pi}$ on the set $\mathbf{S}$ (defined by (2.1)), with respect to which $\mathbf{S}$ has a Boolean structure. $\mathbf{R}_{\pi}$ is defined:

$$
\mathbf{R}_{\pi}\left(s, s^{\prime}, \text { iff } \pi\left(s \& \sim s^{\prime}, \mathbf{C}\right)=0=\pi\left(s^{\prime} \& \sim s, \mathbf{C}\right),\right.
$$

for all $s, s^{\prime} \in \mathbf{S}$. $\mathbf{R}_{\pi}$ is an equivalence relation on $\mathbf{S}$, by (2.1) and D2(I). Let $\underline{\&}$ be the binary operation on $\mathbf{S}$ such that $\underline{\&}\left(s, s^{\prime}\right)=s \& s^{\prime}$. Let $\simeq$ be the unary operation on $\mathbf{S}$ such that $\sim(s)=\sim s$. Then from D2 and D1 it follows that:

(2.4) (S, \&, $\simeq$ ) is a Boolean algebra with respect to $\mathbf{R}_{\pi}$.

The proof of (2.4), with inessential differences, is contained in [3]. From (2.4) it follows that, if $R$ is the intersection of all $R_{\pi}$ defined by (2.3), then $(S, R)$ is a sentential calculus. We now define:

(2.5) $\mathbf{T}_{0}=\{s \in \mathrm{L}: \pi(s, \mathrm{C})=1\}$.

By (1.2), $\mathbf{T}_{0}$ is non-empty, and by D1(I), $\mathbf{T}_{0}$ is a subset of S. We now assert:

(2.6) $\left(\mathbf{S}, \mathbf{T}_{0}\right)$ is a consistent Boolean logic with respect to $\mathbf{R}_{\pi}$.

$\mathbf{T}_{0}$ contains exactly the elements of $\mathbf{S}$ which are $\mathbf{R}_{\pi}$-equivalent to $\sim(s \& \sim s)$, where $s \in \mathbf{S}$. Thus $\mathbf{T}_{0}$ is a filter of the Boolean algebra on $\mathbf{S}$, with respect to $\mathbf{R}_{\pi}$. Moreover, if $s, \sim s \in \mathbf{T}_{0}$, then $\pi(s, \mathbf{C})=1=\pi(\sim s, \mathbf{C})$, against D1(IV). Thus (2.6) is true.

The logic $\left(\mathbf{S}, \mathbf{T}_{0}\right)$ is complete, in the sense of $\mathbf{T}_{\mathbf{0}}$ being maximal in $\mathbf{S}$, iff $\pi(s, C) \neq 2$, for all $s \in S$. The intersection of all $\mathbf{T}_{0}$ defined by (2.5) contains exactly the tautologies of $\mathbf{S}$, i.e., the elements of $\mathbf{S}$ mapped to 1 by every homomorphism of the algebra $(S, \&, \sim)$ onto the simple Boolean algebra.

3. Polyadic Interpretations Pragmatic interpretations of $\mathbf{L}$ which induce on an appropriate subset of $L$ the structure of a polyadic Boolean algebra, we shall call polyadic interpretations. It is convenient first to define the concept of an I-interpretation of $L$, the motivation for which will become subsequently evident.

D3. $\Pi$ is an 1 -interpretation of $\mathrm{L}$ iff $\Pi$ is a proto-sentential interpretation of $\mathrm{L}$ and there is a unique element $\exists$ of $\mathrm{L}$, distinct from \& and $\sim$, such that $\mathbf{I}=\left\{i \in \mathbf{L}: \Pi(\exists i p)=d_{0}\right.$ for some $\left.p \in \mathbf{L}\right\}$ and for all $e, e^{\prime}$ in $\mathbf{L}$ and $i$ in $\mathbf{I}$ :

I. $\quad \Pi(\exists \& e)=\Pi(\exists \sim e)=\Pi(\exists \exists e)=d_{2}$.

II. $\quad \Pi\left(e \& i e^{\prime}\right)=\Pi\left(e \sim i e^{\prime}\right)=d_{2}$.

III. $\Pi(e \&)=\Pi(e \sim)=\Pi(e \exists)=d_{2}$.

IV. If e contains only $\&, \sim, \exists$, and $i$, then $\Pi(e)=d_{2}$.

V. If $\Pi(\exists i$ e $i \ldots i \sim e i \ldots i)=d_{0}$ (n occurrences of $\left.i\right)$, then $(\exists i e i \ldots i \sim$ e $i . . i)=d_{2}(m \neq n$ occurrences of $i)$.

VI. I is infinite. 
VII. If $\Pi(\& e \sim e)=d_{0}$, then every occurrence of $i \in \mathbf{I}$ in $e$ is in a part of $e$ of the form $\exists i e^{\prime}$, for some $e^{\prime} \epsilon \mathbf{L}$.

From D3(I) it follows that \&, , $\exists \notin \mathrm{I}$. From D3(IV) it follows that $\exists \notin S$. We now define, for positive integer $n$ :

$$
\mathbf{P}^{n}=\left\{F \epsilon \mathbf{L}: \Pi(\exists i \& F i \ldots i \sim F i \ldots i)=d_{0} \text { for some } i \epsilon \mathbf{I} \text { ( } n\right. \text { occurrences }
$$
of $i)\}$.

(3.2) $\mathbf{K}=\left\{a \in \mathrm{L}: \Pi(\& F a \ldots a \sim F a \ldots a)=d_{0}\right.$ for some $F \in \mathbf{P}^{n}$ (n occurrences of $a)\}$.

Intuitively, $\mathrm{I}$ is a set of individual variables, $\mathrm{P}^{n}$ is a set of predicates of degree $n$, and $\mathbf{K}$ is a set of individual constants; these intuitions will be justified in section 4 . We now assert that by D3(II) and (IV): \&, , $\exists \notin \mathbf{P}^{n}$. And by D3(III): \&, , $\exists \notin K$. Finally, from D3(V) it follows that the sets $\mathbf{P}^{n}$ are pairwise disjoint. The infinitude of $I$ is required in definition (3.11).

As a preliminary to defining the concept of a polyadic interpretation, we define the concept of a proto-polyadic interpretation. Let $\mathbf{P}^{n}$ and $\mathbf{K}$ be defined by (3.1) and (3.2) respectively.

D4. $\Pi$ is a proto-polyadic interpretation of $\mathbf{L}$ iff $\Pi$ is an $\mathbf{1}$-interpretation of $\mathbf{L}$ for some $\mathbf{I}$ and for all $F, t_{1}, \ldots, t_{n}, p, q, e, e^{\prime}$ in $\mathbf{L}$ :

I. If $F \in \mathbf{P}^{n}$ and $t_{1}, \ldots, t_{n} \in \mathbf{I} \cup \mathbf{K}$, then for some $e \in \mathbf{L}, \Pi\left(e \& F t_{1} \ldots t_{n} \sim\right.$ $\left.F t_{1} \ldots t_{n}\right)=d_{0}$.

II. If $\Pi(e \& p \sim p)=d_{0}=\Pi\left(e^{\prime} \& q \sim q\right)$, then $\Pi\left(e^{\prime \prime} \& \& p q \sim \& p q\right)=d_{0}$, for some $e^{\prime \prime} \in \mathrm{L}$.

III. If $\Pi(e \& p \sim p)=d_{0}$, then $\Pi(e \& \sim p \sim \sim p)=d_{0}$.

IV. If $\Pi(e \& p \sim p)=d_{0}$ and $i \epsilon \mathrm{I}$, then $\Pi(e \& \exists i p \sim \exists i p)=d_{0}$.

V. If $\Pi(e \& p \sim p)=d_{0}$, then one of the following conditions holds:

(a) $p=F t_{1} \ldots t_{n}$ for some $F \in \mathbf{P}^{n}$ and $t_{1}, \ldots, t_{n} \in \mathbf{I} \cup \mathbf{K}$.

(b) $p=\& q r$ and $\Pi(e \& q \sim q)=d_{0}=\Pi(e \& r \sim r)$ for some $e, q, r \in \mathrm{L}$.

(c) $p=\sim q$ and $\Pi(e \& q \sim q)=d_{0}$ for some $e, q \in \mathrm{L}$.

(d) $p=\exists i q$ and $\Pi(e \& q \sim q)=d_{0}$ for some $e, q \in \mathrm{L}$.

If $\Pi$ is a proto-polyadic interpretation of $L$, we define:

$$
\mathbf{Q}=\left\{p \in \mathbf{L}: \Pi(e \& p \sim p)=d_{0} \text { for some } e \epsilon \mathbf{L}\right\}
$$

Then by D4:

(3.4) $\mathbf{Q}$ is the smallest subset of $\mathbf{L}$ such that:

(a) $F t_{1} \ldots t_{n} \in \mathbf{Q}$, if $F \in \mathbf{P}^{n}$ and $t_{1}, \ldots, t_{n} \in \mathbf{I} \cup \mathbf{K}$.

(b) \&pq, $p, \exists i p \in \mathbf{Q}$, if $p, q \in Q$ and $i \in \mathbf{I}$.

The set $\mathbf{S}$ defined by (2.1) is a proper subset of $\mathbf{Q}$, since one may let $e$ in (3.3) be the empty expression; properness of $S$ follows from D3(VII) and $\mathrm{D} 4(\mathrm{I})$. The set $\mathbf{P}=\mathbf{P}^{1} \cup \mathbf{P}^{2} \cup \ldots$ defined by (3.1) is non-empty, by (3.4).

For perspicuity we introduce the following definitions: " $(p) \&(q)$ ") for "\& $p q "$, " " $(p)$ " for " $\sim p$ ", " $(\exists i)(p)$ ", for " $\exists i p$ ", and " $F\left(t_{1}, \ldots, t_{n}\right)$ " for " $F t_{1} \ldots t_{n}$ ", whenever $p, q \in \mathbf{Q} ; i \in \mathbf{I} ; F \in \mathbf{P}^{n} ; t_{1}, \ldots, t_{n} \in \mathbf{I} \cup \mathbf{K}$. We shall continue to omit brackets when this convenience is unambiguous.

We shall define a polyadic interpretation as a proto-polyadic interpre- 
tation which induces a polyadic Boolean algebra on the set $\mathbf{Q}$. In virtue of (3.4), the elements of $Q$ may be regarded as (first order) predicate formulas by labelling the sets $\mathbf{P}^{1}, \mathbf{P}^{2}, \ldots, \mathrm{I}, \mathrm{K},\{\&, \sim, \exists\}$ as sets of predicates of degree $1,2, \ldots$, individual variables, individual constants, and appropriate logical constants, respectively. We shall show that in the pragmatically induced polyadic algebra on $Q$, there is a representation of each of these sets which justifies their being labelled as syntactic categories in the above manner. We now outline this representation, indicating for each of the above syntactic categories the theorem (to be proved subsequently) expressing its algebraic counterpart.

The expressions $F \in \mathbf{P}^{n}$ are representable as predicates (Theorem 2), and $I$ is the set of variables, of the polyadic algebra induced pragmatically on $\mathbf{Q}$ (Theorem 1). Expressions of the form $\exists i$, with $i \epsilon \mathrm{I}$, are representable as quantifiers of this algebra (Theorem 1). Expressions of $\mathbf{K}$ are representable as individual constants of the algebra (Theorem 3). The expressions \& and $\sim$ are representable as Boolean operations of the algebra (Lemma (4.2)).

Henceforth we shall refer to the elements of $Q$ as (predicate) formulas (of first order), and to the elements of $\mathbf{P}, \mathbf{I}, \mathbf{K},\{\&, \sim, \exists\}$ in the grammatical terminology indicated above. We shall also require the concepts of free and bound variables of formulas of $Q$, which may be defined in the usual way: an occurrence of a variable $i$ in a formula $p$ is defined to be bound if it is part of an occurrence of some formula $\exists i q$ in $p$; otherwise the occurrence of $i$ is free. If $p$ has no free variables, it is closed; otherwise it is open.

The task of defining polyadic interpretations is facilitated by introducing the following notation for the formulas of $\mathbf{Q}$. The elements of I may be ordered lexicographically, in the customary way. Let $J=\left\{i_{1}, \ldots, i_{n}\right\}$ be any finite subset of $\mathrm{I}$, in lexicographic order. We then define:

$$
\exists J p=\exists i_{1} \ldots i_{n} p \text {. }
$$

Let $H$ be the set of free variables of $p . H$ is finite since $p$ is a finite sequence of elements of $L_{0}$. We then define, for all $J \subset I$ :

$$
\exists(J)(p)=\left\{\begin{array}{l}
\exists_{J} p \text { if } J \text { is finite. } \\
\exists_{J \cap H} p \text { otherwise. }
\end{array}\right.
$$

It is useful to abbreviate " $\exists(I)(p)$ " as " $\exists(p)$ ". Then with the customary conventions about omitting brackets, if $H$ is the set of free variables of $p$, $\exists(H) p=\exists p$. From (3.6) it follows that, where $\Lambda$ is the empty set:

(3.7) $\exists(\Lambda) p=p$.

We may now define the concept of a polyadic interpretation, provided we apply to the formulas of $Q$ the concept of relettering of bound variables in the sense of alphabetic variation [2]. If $\exists i p$ and $\exists j q$ are formulas of $Q$, then they are defined to be immediate alphabetic variants if $q$ is like $p$ except for containing free $j$ where and only where $p$ contains free $i$. In the following definition the substitutivity notation (2.2) is employed. 
D5. $\Pi$ is a polyadic interpretation of $L$ iff $\Pi$ is a proto-polyadic interpretation of $\mathbf{L}$ and for all $p, q, r \in \mathbf{Q} ; J \subset \mathbf{I} ; e \in \mathbf{L} ; u \in \mathbf{U} ; w \in \mathbf{W} ; c \subset \mathbf{C}$ :

I. $\Pi(\exists(p \& \sim \exists(J) p \& q))=d_{0}$.

II. If $\Pi(\exists(p \& \sim q))(u, w, c)=0=\Pi(\exists(q \& \sim r))(u, w, c)$, then $\Pi(\exists(p \& \sim r))$ $(u, w, c)=0$.

III. If $\Pi(\exists(p \& \sim q))(u, w, c)=0$, then $\Pi(\exists((p \& r) \& \sim(q \& r)))(u, w, c)=0$.

IV. $\Pi(e(p))=\Pi(e(\sim \sim p))=\Pi(e(p \& p))$.

V. $\Pi(e(p \& q))=\Pi(e(q \& p))$.

VI. $\Pi(e(p \&(q \& r)))=\Pi(e((p \& q) \& r))$.

VII. If $\Pi(\exists(p \& \sim q))(u, w, c)=0$, then $\Pi(\exists p)(u, w, c)=\Pi(\exists(p \& q))(u, w, c)$.

VIII. $\Pi(e(\exists(J)(p \& \exists(J) q)))=\Pi(e(\exists(J) p \& \exists(J) q))$.

IX. If $\Pi(\exists(p \& \sim q))(u, w, c)=0$, then $\Pi(\exists(\exists(J) p \& \sim \exists(J) q))(u, w, c)=0$.

$\mathrm{X}$. If $J$ contains no free variables of $p$, then $\Pi(e(p))=\Pi(e(\exists(J) p))$.

XI. $\Pi(e(\exists i \exists j p))=\Pi(e(\exists j \exists i p))$, if $i, j \in \mathbf{I}$.

XII. If $p$ and $q$ are immediate alphabetic variants, then $\Pi(e(p))=\Pi(e(q))$.

Let $\pi$ be the core of a polyadic interpretation $\Pi$ of $L$. Then we have the following consequences of D5 (Roman numerals refer to conditions of D5 unless otherwise indicated). By I, (3.7), V, and VI, for all $c \subset \mathbf{C}, p \in \mathbf{Q}$ :

(3.8) $\pi(\exists(p \& \sim p), c)=0$.

(3.9) If $\pi(\exists p, c)=0$, then $\pi(\exists(p \& q), c)=0$,

for all $p, q \in \mathbf{Q}$. For proof of (3.9) we note that by hypothesis and IV, $\pi(\exists(p \& \sim \sim p), c)=0$. Then by II, $\pi(\exists((p \& q) \& \sim(\sim p \& q)), c)=0$. Then by VII and I: $\pi(\exists(p \& q), c)=\pi(\exists(p \& q \& \sim p \& q), c)=0$.

From (3.7) and (3.8) one may establish the useful proposition:

(3.10) $\pi(\exists((p \& q) \& \sim p), c)=0=\pi(\exists((p \& q) \& \sim q), c)$.

The theory of polyadic interpretations includes the theory of sentential interpretations. For if $\Pi$ is a polyadic interpretation of $L$, then $\Pi$ is a proto-sentential interpretation of $L$ and there is $\mathbf{S} \subset \mathbf{Q}$, with $\mathbf{S}$ defined by (2.1) and $Q$ by (3.3). Then by D5(I - VI), $\Pi$ and $S$ satisfy the definiens of D2, so that $\Pi$ is a sentential interpretation of $L$. Then $(S, \underline{\&}, \simeq$ ) is a Boolean algebra with respect to $R_{\pi}$, defined by (2.3), and all the Boolean properties of $\mathbf{S}$ which are established in section 2 are also forthcoming. By (3.8) and D3(VII), $\mathbf{S}$ is the set of closed formulas of $\mathbf{Q}$.

We shall conclude this section by formulating a notation for expressing substitution operations on free variables of formulas $p \in \mathbf{Q}$, which will be useful in characterizing the pragmatically induced polyadic structure on $\mathbf{Q}$. Let $\tau$ be a transformation on the set $\mathbf{I}$ of variables. Let $i_{1}, \ldots, i_{m}$ be the distinct free variables of $p \in Q$, in lexicographic order, such that $\tau i_{k}$ is not free for $i_{k}$ in $p(1 \leq k \leq m)$; i.e., there is an occurrence of $i_{k}$ in $p$ which is part of some formula $\exists \tau i_{k} q$ in $p$. Let $j_{1}, \ldots, j_{m}$ be the lexicographically first $m$ variables of $\mathbf{I}$ which are not in $p$ nor among the variables $\tau i$, for $i$ free in $p$. Such $j$ always exist, by the infinitude of 1 . With respect to the above $i_{k}, j_{k} \in \mathbf{I}$, we define: 
(3.11) $p_{\tau}=\left\{\begin{array}{l}\text { the formula obtained from } p \text { by relettering each } \tau i_{k} \\ \text { which occurs bound in } p \text { with } j_{k}\end{array}\right.$

for all $p \in \mathbf{Q}$ and transformations $\tau$ on I. If $p$ is closed, there are no $\tau i_{k}$, so that $p_{\tau}=p$.

With respect to the operation of substituting terms for free variables of a formula $p$, it is convenient to express $p$ as $p\left(i_{1}, \ldots, i_{n}\right)$, where $i_{1}, \ldots, i_{n}$ are the free variables of $p$, in any order; if $p$ has no free variables, then for the sake of convenience, the $i_{1}, \ldots, i_{n}$ may be chosen in any way, so that we may apply this notation to all formulas of Q. If $\tau$ is a transformation on $\mathrm{I}$, then $p\left(\tau i_{1}, \ldots, \tau i_{n}\right)$ expresses the result of substituting $\tau i$ for free $i$ in $p=p\left(i_{1}, \ldots, i_{n}\right)$. Thus if $p$ is closed, $p\left(\tau i_{1}, \ldots, \tau i_{n}\right)=p\left(i_{1}, \ldots, i_{n}\right)=p$, for all $i_{1}, \ldots i_{n} \in \mathbf{I}$.

We now define a substitution operation $S(\tau)$ on the $p \in \mathbf{Q}$, for each transformation $\tau$ on I, whose value $S(\tau) p$ is the formula obtained from $p_{\tau}$ by substituting $\tau i$ for free $i$ in $p_{\tau}$. Expressing $p$ as $p\left(i_{1}, \ldots, i_{n}\right)$, according to the above substitution notation, we define:

(3.12) $\mathrm{S}(\tau)\left(p\left(i_{1}, \ldots, i_{n}\right)\right)=p_{\tau}\left(\tau i_{1}, \ldots, \tau i_{n}\right)$.

If $p$ is closed, $\mathrm{S}(\tau) p=p\left(\tau i_{1}, \ldots, \tau i_{n}\right)=p\left(i_{1}, \ldots, i_{n}\right)=p$, for all $i_{1}, \ldots, i_{n} \in \mathbf{I}$. From definition (3.12) it follows that:

(3.13) $\mathrm{S}(\tau) \sim p=\sim \mathrm{S}(\tau) p$.

(3.14) $\mathrm{s}(\delta) p=p$,

where $\delta$ is the identity transformation on $I$.

(3.15) $S(\sigma) \exists(J) p=S(\tau) \exists(J) p$, if $\sigma=\tau$ on $\mathbf{I}-J$.

Let $a \in \mathrm{K}, p \in \mathbf{Q}, J \subset \mathrm{I}$. Then we define:

$$
p\left(a_{J}\right)=\left\{\begin{array}{l}
\text { the formula obtained by replacing with a all } \\
\text { variables in } J \text { which occur free in } p .
\end{array}\right.
$$

If $p$ is closed, then $p\left(a_{J}\right)=p$, for all $J \subset \mathrm{I}$. It also follows from (3.16) that, for all $J, H \subset$ I:

(3.17) $(p \& q)\left(a_{J}\right)=p\left(a_{J}\right) \& q\left(a_{J}\right)$.

(3.18) $\quad(\sim p)\left(a_{J}\right)=\sim\left(p\left(a_{J}\right)\right)$.

(3.19) $p\left(a_{\Lambda}\right)=p$.

(3.20) $p\left(a_{J \cup H}\right)=\left(p\left(a_{J}\right)\right)\left(a_{H}\right)$.

(3.21) $(\exists(H) p)\left(a_{J}\right)=\exists(H)\left(p\left(a_{J-H}\right)\right)$.

4. Polyadic Boolean Algebras of Predicate Formulas Let $\Pi$ be a polyadic interpretation of $L, \pi$ its core, and let $Q$ be defined by (3.3), throughout this section. Then there is a natural equivalence relation $E_{\pi}$ on $Q$, with respect to which $\mathbf{Q}$ has a polyadic Boolean structure. $\mathbf{E}_{\pi}$ is defined, for all $p, q \in \mathbf{Q}$ :

(4.1) $\mathbf{E}_{\pi}(p, q)$ iff $\pi(\exists(p \& \sim q), \mathrm{C})=0=\pi(\exists(q \& \sim p), \mathrm{C})$.

$E_{\pi}$ is an equivalence relation on $Q$, by (3.8) and D5(II). The statement and proof of the proposition that there is a polyadic Boolean algebra induced on 
$Q$ by $\Pi$ requires the following lemma. Let $\underline{\&}(p, q)=p \& q$ and $\sim(p)=\sim p$, for $p, q \in \mathbf{Q}$. Let $\mathbf{E}_{\pi}$ be defined by (4.1).

(4.2) $(\mathbf{Q}, \underline{\&}, \simeq)$ is a Boolean algebra with respect to $\mathbf{E}_{\pi}$.

Proof: (Roman numerals refer to conditions of D5, unless indicated otherwise.) $Q$ is closed under the operations \& and $\simeq$, by (3.4). By (3.8), $\pi(\exists((p \& q) \& \sim(p \& q)), \mathrm{C})=0$. Then by $\mathrm{V}, \pi(\exists((p \& \bar{q}) \& \sim(\bar{q} \& p)), \mathrm{C})=0$. In this way one shows:

(i) $\mathbf{E}_{\pi}(p \& q, q \& p)$.

In the same way one may show:

(ii) $\mathrm{E}_{\pi}(p \&(q \& r),(p \& q) \& r)$.

We next show:

(iii) if $\mathbf{E}_{\pi}(p, q)$, then $\mathbf{E}_{\pi}(\sim p, \sim q)$.

By hypothesis, $\pi(\exists(p \& \sim q), \mathrm{C})=0$. Then by IV and $\mathrm{V}, \pi(\exists(\sim q \& \sim \sim p), \mathrm{C})=0$. In this way one obtains (iii). We next assert:

(iv) if $\mathbf{E}_{\pi}(p, q)$, then $\mathbf{E}_{\pi}(p \& r, q \& r)$,

which follows directly from III. We next assert:

(v) if $\mathrm{E}_{\pi}(p \& \sim q, r \& \sim r)$, then $\mathrm{E}_{\pi}(p, p \& q)$.

By hypothesis, $\pi(\exists((p \& \sim q) \& \sim(r \& \sim \gamma)), \mathrm{C})=0$. Then by VII and (3.8): $\pi(\exists(p \& \sim q), \mathrm{C})=\pi(\exists((p \& \sim q) \&(r \& \sim r)), \mathrm{C})=0$. Then by III, IV, V: $\pi(\exists((p \&$ p) $\& \sim(q \& p)), \mathrm{C})=\pi(\exists(p \& \sim(p \& q)), \mathrm{C})=0$. This with (3.10) gives (V). We next show:

(vi) if $\mathbf{E}_{\pi}(p, p \& q)$, then $\mathbf{E}_{\pi}(p \& \sim q, r \& \sim r)$.

By hypothesis, $\pi(\exists(p \& \sim(p \& q)), \mathrm{C})=0$. Then by (3.10), II, and (3.9): $\pi(\exists(p \& \sim q), \mathrm{C})=0=\pi(\exists((p \& \sim q) \& \sim(r \& \sim \gamma)), \mathrm{C})$. For all $p, q, r \in \mathrm{Q}: \pi(\exists((r \& \sim$ $r) \& \sim(p \& \sim q)), \mathrm{C})=0$. Thus (vi) is true; and by (i)-(vi), (4.2) is proved.

Let $\underline{S}$ be the mapping from transformations $\tau$ on $I$ to transformations $S(\tau)$, on $Q$, defined by (3.12). Let $\exists$ be the mapping from subsets $J$ of $I$ to transformations $\exists(J)$ on $Q$, defined by (3.6).

Theorem 1. $(\mathbf{Q}, \mathrm{I}, \underline{\mathrm{S}}, \underline{\exists})$ is a locally finite polyadic Boolean algebra of infinite degree, with respect to $\mathrm{E}_{\pi}$.

Proof: We first show that $(Q, I ; \underline{S})$ is a transformation algebra with respect to $\mathbf{E}_{\pi}$. By (3.8) and (3.13):

(S1) $\mathrm{E}_{\pi}(\mathrm{S}(\tau) \sim p, \sim \mathrm{S}(\tau) p)$.

We next observe that $S(\tau)(p \& q)$ and $S(\tau) p \& S(\tau) q$ are alphabetic variants, i.e., intertransformable by a succession of immediate alphabetic variations. For:

$\mathrm{S}(\tau) p\left(i_{1}, \ldots, i_{m}\right) \& \mathrm{~S}(\tau) q\left(i_{m+1}, \ldots, i_{m+n}\right)=p_{\tau}\left(\tau i_{1}, \ldots, \tau i_{m}\right) \& q_{\tau}\left(\tau i_{m+1}, \ldots, \tau i_{m+n}\right)$; $\mathrm{S}(\tau)(p \& q)\left(i_{1}, \ldots, i_{m}, i_{m+1}, \ldots, i_{m+k}\right)=(p \& q)_{\tau}\left(\tau i_{1}, \ldots, \tau i_{m+k}\right)$; 
where $i_{m+1}, \ldots, i_{m+k}$ are the free variables of $q$ distinct from those of $p$. Thus $\mathrm{S}(\tau)(p \& q)$ and $\mathrm{S}(\tau) p \& \mathrm{~S}(\tau) q$ differ at most in relettering of bound variables, as follows. Any relettering in transforming $p \& q$ to $S(\tau)(p \& q)$ is either identical to the corresponding relettering in transforming either $p$ to $\mathrm{S}(\tau) p$ or $q$ to $\mathrm{S}(\tau) q$, or else employs a variable which does not occur in $\mathrm{S}(\tau) p$ or $\mathrm{S}(\tau) q$. Then $\mathrm{S}(\tau)(p \& q)$ may be obtained from $\mathrm{S}(\tau) p \& \mathrm{~S}(\tau) q$ by successive reletterings with variables not in the latter, so that the two formulas are alphabetic variants. Then by (3.8) and repeated applications of XII:

$$
\mathbf{E}_{\pi}(\mathrm{S}(\tau)(p \& q), \mathrm{S}(\tau) p \& \mathrm{~S}(\tau) q)
$$

By (S1) and (S2), the transformations $S(\tau)$ on $Q$ are Boolean endomorphisms with respect to $E_{\pi}$. If $\delta$ is the identity transformation on $I$, then by (3.14):

$\mathbf{E}_{\pi}(p, s(\delta) p)$

$\mathrm{S}(\sigma \tau) p$ and $\mathrm{S}(\sigma) \mathrm{S}(\tau) p$ are alphabetic variants, where $\sigma$ and $\tau$ are transformations on I. For $S(\sigma \tau) p\left(i_{1}, \ldots, i_{n}\right)=p_{\sigma \tau}\left(\sigma \tau i_{1}, \ldots, \sigma \tau i_{n}\right)$, and $\mathrm{S}(\sigma) \mathrm{S}(\tau) p\left(i_{1}, \ldots, i_{n}\right)=\mathrm{S}(\sigma) p_{\tau}\left(\tau i_{1}, \ldots, \tau i_{n}\right)=\left(p_{\tau}\right)_{\sigma}\left(\sigma \tau i_{1}, \ldots, \sigma \tau i_{n}\right)$. Now $p$ is an alphabetic variant of $p_{\sigma \tau}$, since the latter is obtained by relettering distinct bound variables of $p$ with distinct variables not in $p$. For the same reason, $p_{\tau}$ is an alphabetic variant of $p$; and $\left(p_{\tau}\right)_{\sigma}$ is an alphabetic variant of $p_{\tau}$, and hence of $p_{\sigma \tau}$. If any relettering with $\sigma \tau i$ (for $i$ free in $p$ ) is required in the above alphabetic variation of $p_{\sigma \tau}$ and $\left(p_{\tau}\right)_{\sigma}$ via $p_{\tau}$ and $p$, then there is an alternative relettering involving some variable distinct from such $\sigma \tau i$, so that there is an alphabetic variation of $p_{\sigma r}$ and $\left(p_{\tau}\right)_{\sigma}$ which does not involve relettering with $\sigma \tau i$. This is also an alphabetic variation of $p_{\sigma \tau}\left(\sigma \tau i_{1}, \ldots, \sigma \tau i_{n}\right)$ and $\left(p_{\tau}\right)_{\sigma}\left(\sigma \tau i_{1}, \ldots, \sigma \tau i_{n}\right)$. Thus by XII:

$$
\mathbf{E}_{\pi}(S(\sigma \tau) p, S(\sigma) S(\tau) p) \text {. }
$$

We next show:

(S5) if $\mathbf{E}_{\pi}(p, q)$, then $\mathbf{E}_{\pi}(\mathrm{S}(\tau) p, \mathrm{~S}(\tau) q)$.

By hypothesis of (S5), $\pi(\exists(J)(p \& \sim q), \mathrm{C})=0$, where $J$ is the set of free variables of $p \& \sim q$. Now $\exists(\tau J) S(\tau)(p \& \sim q)$ is an alphabetic variant of $\exists(J)(p \& \sim q)$. For any relettering in transforming $p \& \sim q$ to $(p \& \sim q)_{\tau}$ requires variables distinct from those of $p \& \sim q$ and from those in $\tau J$. Then by XII, $\pi(\exists(S(\tau)(p \& \sim q)), \mathrm{C})=0$. Now $S(\tau)(p \& \sim q)$ is an alphabetic variant of $\mathrm{S}(\tau) p \& \mathrm{~S}(\tau) \sim q=\mathrm{S}(\tau) p \& \sim \mathrm{S}(\tau) q$, as shown in the proof of (S2). Then $\pi(\exists(S(\tau) p \& \sim S(\tau) q), C)=0$. In this way, (S5) is shown.

By $(\mathrm{S} 1)$ - (S5), $(\mathbf{Q}, \mathbf{I}, \underline{\mathrm{S}})$ is a transformation algebra with respect to $\mathbf{E}_{\pi}$. We next show that $(\mathbf{Q}, \mathbf{I}, \underline{\exists})$ is a quantifier algebra with respect to $\mathbf{E}_{\pi}$. By (3.7):

( $\exists 1) \mathbf{E}_{\pi}(p, \exists(\Lambda) p)$.

By repeated applications of XI, for all $J, H \subset \mathrm{I}$ :

$\mathbf{E}_{\pi}(\exists(J \cup H) p, \exists(J) \exists(H) p)$. 
By VIII, for $J \subset$ I:

( $\exists 3) \mathbf{E}_{\pi}(\exists(J)(p \& \exists(J) q), \exists(J) p \& \exists(J) q)$.

We next observe that, by I, IV-VI: $\pi(\exists(p \& \sim \exists(J) p), \mathrm{C})=0$. Then by III, $\pi(\exists(p \& \sim(p \& \exists(J) p)), C)=0$. This with $(3.10)$ proves:

( $\exists 4) \quad \mathbf{E}_{\pi}(p, p \& \exists(J) p)$.

Let $\mathbf{E}_{\pi}\left(p_{0}, q \& \sim q\right)$ for some $q \in \mathbf{Q}$. Then for all $J \subset \mathbf{I}$ :

(ヨ5) $\mathbf{E}_{\pi}\left(p_{0}, \exists(J) p_{0}\right)$.

Since $\mathrm{E}_{\pi}\left(p_{0}, q \& \sim q\right)$, we have $\pi\left(\exists\left(p_{0} \& \sim(q \& \sim q)\right), \mathrm{C}\right)=0$. Then by VII: $\pi\left(\exists p_{0}, \mathrm{C}\right)=\pi\left(\exists\left(p_{0} \& q \& \sim q\right), \mathrm{C}\right)=0=\pi\left(\exists(H) p_{0}, \mathrm{C}\right)$, where $H$ is the set of free variables of $p_{0}$. Then by $\mathrm{X}, \mathrm{XI}$, for all $J \subset \mathrm{I}: \pi\left(\exists(J) \exists(H) p_{0}, \mathrm{C}\right)=0=$ $\pi\left(\exists(H) \exists(J) p_{0}, \mathrm{C}\right)$. Then by $(3.9): \pi\left(\exists(H)\left(\exists(J) p_{0} \&\left(\sim p_{0}\right)\right), \mathrm{C}\right)=0$. This with I gives $(\exists 5)$.

( $\exists 6)$ if $\mathbf{E}_{\pi}(p, q)$, then $\mathbf{E}_{\pi}(\exists(J) p, \exists(J) q)$,

for all $J \subset I$. ( $\exists 6)$ follows immediately from $\mathrm{XX}$. By $(\exists 1)-(\exists 6),(Q, I, \exists)$ is a quantifier algebra with resepct to $\mathbf{E}_{\pi}$. For proof that $(\mathbf{Q}, \mathbf{I}, \underline{S}, \underline{\exists})$ is a polyadic algebra, it remains to show that $\underline{\exists}$ and $\underline{S}$ are related in the proper way. If $J \subset I$ and $\sigma=\tau$ on $\mid-J$, then:

$$
\mathbf{E}_{\pi}(S(\sigma) \exists(J) p, S(\tau) \exists(J) p),
$$

by (3.15). Finally, if $\tau$ is one-one on $\tau^{-1} J$, then:

$$
E_{\pi}\left(\exists(J) S(\tau) p, S(\tau) \exists\left(\tau^{-1} J\right) p\right) .
$$

For these formulas are alphabetic variants. $\exists(J) S(\tau) p$ is $\exists(J) p_{\tau}\left(\tau i_{1}, \ldots, \tau i_{n}\right)$, with free variables $\tau i \xi J$; and $S(\tau) \exists\left(\tau^{-1} J\right) p$ is $\left(\exists\left(\tau^{-1} J\right) p\right)_{\tau}\left(\tau i_{1}, \ldots, \tau i_{n}\right)$, with free variables $\tau i$ such that $i \notin \tau^{-1} J$. Thus the formulas of ( $\left.\exists \mathrm{S} 2\right)$ have the same free variables. Since $\tau$ is one-one on $\tau^{-1} J, \tau$ introduces no reflexivity into $S(\tau) p$ which may be obscured in $S(\tau) \exists\left(\tau^{-1} J\right) p$ by binding such variables in $p$ before they are transformed by $\tau$. Thus $\exists(J)$ and $\exists\left(\tau^{-1} J\right)$ contain the same number of quantifiers. Each distinct $\exists(J)$-binding in $\exists(J) S(\tau) p$ may be relettered with some distinct variable not in $p$ nor $\exists(J) S(\tau) p$ nor $S(\tau) \exists\left(\tau^{-1} J\right) p$; the resulting formula is an alphabetic variant of the formulas of ( $\exists S 2$ ).

Thus $(\mathbf{Q}, \mathbf{I}, S, \exists)$ is a polyadic algebra with respect to $\mathbf{E}_{\pi}$. Let $p \in \mathbf{Q}$ and $J$ be the set of free variables of $p$. Then by (3.6) and $(3.7): \exists(1-J) p=$ $\exists((I-J) \cap J) p=\exists(\Lambda) p=p$. Then $\mathbf{E}_{\pi}(p, \exists(I-J) p)$ : each $p \in \mathbf{Q}$ has the set of its free variables as finite support. $(Q, I, \underline{S}, \underline{\exists})$ is therefore locally finite. The proof of Theorem 1 is complete.

Let $Q^{*}$ be the subset of $Q$ whose elements contain no individual constants $a \in \mathbf{K}$. If $\mathbf{K}$ is empty, then $\mathbf{Q}=\mathbf{Q}^{*} . \mathbf{Q}^{*}$ is closed under the operations $\&$ and $\simeq$ of (4.2). Moreover, if $p \in \mathbf{Q}^{*}$, then $S(\tau) p, \exists(J) p \in \mathbf{Q}^{*}$.

Corollary (Theorem 1$)$. (Q*, $\mathbf{I}, \underline{\mathbf{S}}, \underline{\exists})$ is a polyadic subalgebra of $(\mathbf{Q}, \mathbf{I}, \underline{\mathbf{S}}, \underline{\exists})$, with respect to $\mathbf{E}_{\pi}$. 
Let the set $\mathbf{S}$ be defined by $(2.1)$. $\mathrm{S}$ is closed under the operations $\underline{\&}$ and $\simeq$ of (4.2). Then we have:

(4.3) $(\mathbf{S}, \underline{\&}, \underline{\sim})$ is a Boolean subalgebra of $(\mathbf{Q}, \underline{\&}, \underline{\sim})$ with respect to $\mathbf{E}_{\pi}$.

Let the sets $\mathbf{P}^{n}$ be defined by (3.1). If the mapping $\left(i_{1}, \ldots, i_{n}\right) \rightarrow$ $F i_{1} \ldots i_{n}$, for $\left(i_{1}, \ldots, i_{n}\right) \in \mathbf{I}^{n}$ and $F \in \mathbf{P}^{n}$, is a predicate of the polyadic algebra on $\mathbf{Q}$, we shall say that the expression $F \in \mathbf{P}^{n}$ is representable by this predicate.

Theorem 2. The expressions $F \in \mathbf{P}^{n}$ are representable by predicates of the polyadic algebra on $\mathbf{Q}$.

Proof: Let $F \in \mathbf{P}^{n}$. Then for each $n$-tuple of variables $\left(i_{1}, \ldots, i_{n}\right) \in \mathbf{I}^{n}$, there is a formula $F i_{1} \ldots i_{n} \in \mathbf{Q}$, by (3.4), and moreover:

$$
\mathbf{E}_{\pi}\left(\mathrm{S}(\tau) F i_{1} \ldots i_{n}, F \tau i_{1} \ldots \tau i_{n}\right)
$$

for all transformations $\tau$ on I. By $(3.11),\left(F i_{1} \ldots i_{n}\right)_{\tau}=F i_{1} \ldots i_{n}$. Then by (3.12) the formulas of (4.4) are identical. Thus the mapping $\left(i_{1}, \ldots, i_{n}\right) \rightarrow$ $F i_{1} \ldots i_{n}$ is a predicate of the polyadic algebra on $Q$, and Theorem 2 is thereby proved.

Let the set $K$ be defined by (3.2). If $a \in \mathbf{K}$ there is a mapping from subsets $J$ of $I$ to transformations $a_{J}$ on $\mathbf{Q}$ such that the value of $a_{J}$ at $p \in \mathbf{Q}$ is $p\left(a_{J}\right)$, defined by (3.16). If this mapping from subsets $J$ of $I$ to transformations $a_{J}$ on $\mathbf{Q}$ is a constant of the polyadic algebra on $\mathbf{Q}$, we shall say that the expression $a$ is representable by this constant.

Theorem 3. The expressions $a \in \mathbf{K}$ are representable by constants of the polyadic algebra on $\mathbf{Q}$.

Proof: By (3.17) and (3.18), each mapping $a_{J}: p \rightarrow p\left(a_{J}\right)$ is a Boolean endomorphism on $Q$ with respect to $E_{n}$. By (3.19)-(3.21):

(C1) $\mathrm{E}_{\pi}\left(p, p\left(a_{\Lambda}\right)\right)$.

(C2) $\mathrm{E}_{\pi}\left(p\left(a_{J \cup H}\right),\left(\left(p\left(a_{J}\right)\right)\left(a_{H}\right)\right)\right.$.

(C3) $\mathbf{E}_{\pi}\left((\exists(H) p)\left(a_{J}\right), \exists(H)\left(p\left(a_{J-H}\right)\right)\right)$.

We next show:

(C4) $\mathbf{E}_{\pi}\left(\exists(J)\left(p\left(a_{H}\right)\right),(\exists(J-H) p)\left(a_{H}\right)\right)$.

If $p=p\left(i_{1}, \ldots, i_{n}\right)$, then $\exists(J)\left(p\left(a_{H}\right)\right)=\exists(J) p\left(t_{1}, \ldots, t_{n}\right)$, where $t_{k}=a$ if $i_{k} \in H$, and $t_{k}=i_{k}$ otherwise; and $(\exists(J-H) p)\left(a_{H}\right)=\exists(J-H) p\left(u_{1}, \ldots, u_{n}\right)$, where $u_{k}=a$ if $i_{k} \in H$, and $u_{k}=i_{k}$ otherwise $(1 \leq k \leq n)$. Then $t_{k}=u_{k}$. For let $t_{k}=a$. Then $i_{k} \in H$, and $u_{k}=a=t_{k}$. Now let $t_{k}=i_{k}$. Then $i_{k} \notin H$, and $u_{k}=i_{k}=t_{k}$. We next show that $i_{k}$ is bound in both or neither of the formulas of (C4). Let $i_{k}$ be bound in $\exists(J)\left(p\left(a_{H}\right)\right)$. Then $i_{k} \in J-H$, and is bound in $(\exists(J-H) p)\left(a_{H}\right)$. The converse is obvious. Thus the formulas of (C4) may differ only in vacuous prenex quantifiers. Then by $\mathrm{X}$ and $\mathrm{XI},(\mathrm{C} 4)$ is true.

(C5) $\mathbf{E}_{\pi}\left((S(\tau) p)\left(a_{J}\right), S(\tau)\left(p\left(a_{\tau}-1_{J}\right)\right)\right)$.

The formulas of $(\mathrm{C} 5)$ are alphabetic variants. For $\left(S(\tau) p\left(i_{1}, \ldots, i_{n}\right)\right)\left(a_{J}\right)=$ 
$\left(p_{\tau}\left(\tau i_{1}, \ldots, \tau i_{n}\right)\right)\left(a_{J}\right)=p_{\tau}\left(t_{1}, \ldots, t_{n}\right)$, where $t_{k}=\tau i_{k}$ if $\tau i_{k} \not J$, and $t_{k}=a$ other wise. And $S(\tau)\left(p\left(a_{\tau^{-1} J}\right)\right)$ is the formula obtained by substituting $\tau_{i}$ for free $i$ in $p\left(a_{\tau-1 J}\right)$, which is of the form $p\left(u_{1}, \ldots, u_{n}\right)$, where $u_{k}=i_{k}$ if $i_{k} \notin \tau^{-1} J$, and $u_{k}=a$ otherwise. Then $S(\tau)\left(p\left(a_{\tau-1}\right)\right)$ is of the form $p\left(a_{\tau}-1_{J}\right)_{\tau}\left(w_{1}, \ldots, w_{n}\right)$, where $w_{k}=\tau i_{k}$ if $u_{k}=i_{k}$, and $w_{k}=a$ otherwise. Now let $w_{k}=\tau i_{k}$, so that $u_{k}=w_{k}$. Then $i_{k} \notin \tau^{-1} J$, so that $\tau i_{k} \notin J$. Then $t_{k}=\tau i_{k}=w_{k}$. In the same way, if $w_{k}=a$, then $t_{k}=a=w_{k}$. Thus the formulas of (C5) may differ only in so far as $p\left(a_{\tau-1}\right)_{\tau}$ is obtained by relettering $p\left(a_{\tau-1}\right)$ with some variable $j \epsilon \tau^{-1} J$ which is pre-empted in relettering $p$ as $p_{\tau}$, because $j$ is free in $p$, but does not occur in $p\left(a_{\tau^{-1} J}\right)$. Then the formulas of (C5) are alphabetic variants; so that (C5) is true by XII. The proof of Theorem 3 is complete.

4.1 Polyadic Logics We continue to let $\pi$ be the core of a polyadic interpretation $\Pi$ of $L$, with $Q$ the set of formulas determined by $\Pi$. We then define the set $\mathbf{T}$ of formulas whose universal closures are accepted under the total evidence $C$.

(4.5) $\mathbf{T}=\{p \in Q: \pi(\exists \sim p, C)=0\}$.

The set $\mathbf{T}$ is a filter, or dual ideal, of the Boolean algebra on $\mathbf{Q}$, with respect to $\mathbf{E}_{\pi}$. For if $p_{1}=\sim(q \& \sim q)$ for some $q \in \mathbf{Q}$, then for all $p \in \mathbf{Q}$ :

(4.6) $p \in \mathbf{T}$ iff $\mathbf{E}_{\pi}\left(p, p_{1}\right)$,

which is an abbreviation of:

(4.7) $\pi(\exists \sim p, C)=0$ iff $\pi\left(\exists\left(p \& \sim p_{1}\right), C\right)=0=\pi\left(\exists\left(p_{1} \& \sim p\right), C\right)$.

(4.7) is established as follows. In general we have that, $\pi\left(\exists\left(p \& \sim p_{1}\right), \mathrm{C}\right)=$ 0 . If $\pi(\exists \sim p, \mathrm{C})=0$, then $\pi\left(\exists\left(p_{1} \& \sim p\right), \mathrm{C}\right)=0$. Conversely, if $\pi\left(\exists\left(p_{1} \& \sim p\right), \mathrm{C}\right)=$ 0 , then $\pi(\exists \sim p, \mathrm{C})=\pi\left(\exists\left(\sim p \& \sim p_{1}\right), \mathrm{C}\right)=0$.

$\mathbf{T}$ is the unit element of the polyadic quotient algebra of $\mathbf{Q}$ modulo the congruence E . Let " $\forall(J) p$ " abbreviate " $\sim \exists(J) \sim p$ ". We then assert that the filter $T$ of the algebra on $Q$ is polyadic, that is:

(4.8) if $p \in \mathbf{T}$, then $\forall(J) p, S(\tau) p \in \mathbf{T}$,

for all $p \in \mathbf{Q}, J \subset H$, and transformations $\tau$ on I. If $p \in \mathbf{T}$, then $\pi(\exists(H) \sim p, \mathrm{C})=$ 0 , where $H$ is the set of free variables of $p$, and we have, for all $J \subset \mathbf{I}$ : $\pi(\exists(J) \exists(H) \sim p, \mathrm{C})=0=\pi(\exists(H) \exists(J) \sim p, \mathrm{C})=\pi(\exists \sim \forall(J) p, \mathrm{C})$. We next observe that $\exists \sim S(\tau) p$ is an alphabetic variant of $\exists \sim p$, a fact which may be established as in the proof of (S5). (4.8) is thereby established.

In general, if $(A, I, S, \exists)$ is a polyadic algebra and $M$ is a polyadic ideal of $A$, then $(A, M)$ is defined (solecistically) to be a polyadic logic. We shall employ the dual concept of a polyadic logic $(A, M)$, where $M$ is a dual ideal, or filter, of $\mathbf{A}$.

Theorem 4. $(\mathbf{Q}, \mathbf{T})$ is a consistent polyadic logic with respect to $\mathbf{E}_{\pi}$.

By (4.6) and (4.8), $T$ is a polyadic filter of the algebra on $Q$. For proof that $(Q, T)$ is consistent, let $T_{0}=T \cap S$. Then $T_{0}$ is the unit element of the Boolean quotient algebra on $S / E_{\pi}$. Thus $T_{0}$ is, with respect to $E_{\pi}$, a filter of the Boolean subalgebra $(\mathbf{S}, \underline{\&}, \underline{\sim})$. We then assert a lemma for Theorem 4: 
$\left(\mathbf{S}, \mathbf{T}_{0}\right)$ is a consistent Boolean logic with respect to $\mathbf{E}_{\pi}$.

If $s, \sim s \in \mathbf{T}_{0}$, then $s$ and $\sim s$ are formulas of $\mathbf{Q}$ with no free variables; but then by $\mathrm{D} 5(\mathrm{X}): \pi(\exists \sim s, \mathrm{C})=\pi(\sim s, \mathrm{C})=0=\pi(\exists s, \mathrm{C})=\pi(s, \mathrm{C})$, against $\mathrm{D} 1(\mathrm{~V})$. Thus (4.9) is true, and Theorem 4 follows immediately: if $T=Q$, then $\mathbf{T}_{0}=\mathbf{S}$; thus properness of $\mathbf{T}$ follows from properness of $\mathbf{T}_{0}$.

4.2 Closed Formulas of $\mathbf{Q}$ The set $\mathbf{S}$ of syntactically closed formulas of $\mathbf{Q}$ (formulas with no free variables) does not coincide with the set $\exists(Q)=$ $\left\{p \in \mathbf{Q}: \mathbf{E}_{\pi}(p, \exists p)\right\}$ of algebraically closed formulas of the polyadic algebra on Q. Rather $\mathbf{S}$ is properly included in $\exists(\mathbf{Q})$. That syntactically closed formulas are algebraically closed follows immediately from D3 (VII) and D5 (X). Moreover, if $p \in \mathbf{T}$, then $\mathbf{E}_{\pi}(p, \exists p)$ even if $p \notin \mathbf{S}$; formulas of the form $\sim(q \& \sim q)$ with free variables are in $\mathbf{T}$ but not in $\mathbf{S}$.

$\exists(Q)$ and $S$ are further related in the following way. If $T_{0}$ is maximal in $S$, then for each $s \in \mathbf{S}, \pi(s, C) \neq 2$ : the set $\mathbf{C}$ of conditions is germane for $\mathbf{S}$. The converse is also true: $\pi(s, \mathrm{C})=1$ iff $\pi(\exists \sim s, \mathrm{C})=0$ iff $s \epsilon \mathrm{T}$; and $\pi(s, \mathrm{C})=$ 0 iff $\pi(\exists \sim \sim s, C)=0$ iff $\sim s \epsilon$ T. Thus we have shown:

\section{$\left(\mathbf{S}, \mathbf{T}_{0}\right)$ is complete iff $\mathbf{C}$ is germane for $\mathbf{S}$,}

where completeness of $\left(\mathbf{S}, \mathbf{T}_{0}\right)$ is to be understood as maximality to $\mathbf{T}_{0}$ in $\mathbf{S}$, and germaneness of $C$ for $S$ is to be understood as the proposition that $\pi(s, \mathbf{C}) \neq 2$ for all $s \in \mathbf{S}$. Now the polyadic logic $(\mathbf{Q}, \mathbf{T})$ is complete iff the associated Boolean logic $(\exists(Q), T \cap \exists(Q))$ is complete. As we have seen, $\mathbf{T} \subset \exists(\mathbf{Q})$, so that $\mathbf{T} \cap \exists(\mathbf{Q})=\mathbf{T}$. Then the following relationship holds between $\mathbf{S}$ and $\exists(\mathbf{Q})$ :

(4.11) $\left(\mathbf{S}, \mathbf{T}_{\mathbf{0}}\right)$ is complete iff $(\exists(\mathbf{Q}), \mathbf{T})$ is complete.

If $\mathbf{T}$ is maximal in $\exists(\mathbf{Q})$, then $\mathbf{T}_{0}$ is maximal in $\mathbf{S}$. Conversely, let $p \in \exists(\mathbf{Q})$ and $\mathbf{T}_{0}$ be maximal in $\mathrm{S}$. Then $\pi(\exists(\exists p \& \sim p), \mathrm{C})=0=\pi(\exists p \& \exists \sim p, \mathrm{C})$, by D5(VIII). Then by D1(I), $\exists p \& \exists \sim p \in \mathbf{S}$. Now $\exists p$ and $\exists \sim p$ are syntactically closed formulas and hence elements of S. Then $\pi(\exists p, \mathrm{C}) \neq 2 \neq \pi(\exists \sim p, \mathrm{C})$, by hypothesis of completeness of $\left(\mathbf{S}, \mathbf{T}_{0}\right)$. Then from the fact that $\pi(\exists p \& \exists \sim p, \mathrm{C})=0$, it follows that $\sim p \epsilon \mathbf{T}$ or $p \in \mathbf{T}$.

We conclude this section with the observation that $\mathbf{E}_{\pi}$ restricted to $\mathbf{S}$ coincides with the relation $\mathbf{R}_{\pi}$ defined by (2.3), since polyadic interpretations are also sentential interpretations. For if $s, s^{\prime} \in \mathbf{S}$, then $s \& \sim s^{\prime}$ and $s^{\prime} \& \sim s$ are closed, and by $\mathrm{D} 5(\mathrm{X}): \mathbf{R}_{\pi}\left(s, s^{\prime}\right)$ iff $\mathbf{E}_{\pi}\left(s, s^{\prime}\right)$. We also remark that $T_{0}=T \cap S$ is identical to the set $T_{0}$ defined by (2.5).

5. On Peirce's Concept of Logic as Semiotic We have shown that a polyadic interpretation of $L$ determines uniquely the subsets $Q$ and $Q_{0}=$ $\mathbf{P} \cup \mathbf{I} \cup \mathbf{K} \cup\{\&, \sim, \exists\}$ of $L$, such that there is a polyadic Boolean algebra on $Q$ (Theorem 1), the elements of $Q$ are sequences of elements of $Q_{0}(3.4)$, and the elements of $\mathbf{Q}_{0}$ are represented in the algebra on $\mathbf{Q}$ by algebraic counterparts of the syntactic categories of the predicate calculus (Theorems 1-3). We have, to be sure, used in the pragmatic meta-language of $L$ such concepts as freedom and bondage of variables of expressions of $\mathbf{Q}$. 
Thus what we have shown is that if the users of $L$ employ some concepts equivalent to the above, in the sense that their valuations constitute a polyadic interpretation of $L$, then we may recognize, and express in the pragmatic meta-language of $L$, the fact that such valuations determine the polyadic algebra $(Q, I, \underline{S}, \underline{\exists})$.

We shall not pursue further the grammatical significance of this result, but shall proceed to analyze its semantical significance. If $\Pi$ is a given polyadic interpretation of $L$ and $e \epsilon \mathrm{L}$, then a valuing disposition $(u, w, c, v) \epsilon \Pi(e)$ may be regarded as an interpretant of $e$ in a sense generalized from that in which pointing at the object of $e$ (if pointing were possible) would be an interpretant of $e$. Now such direct ostensive interpretations of signs as pointing are not always possible; and it was the beauty of Peirce's idea that every genuine signification nevertheless involves some interpretant, which it is the task of logic to discover. The theory of polyadic interpretations is suggested as a contribution to this program for logic, restricted to signs of first order languages.

If the objects signified by the logical constants $\exists, \&, \sim$ of the formulas of $\mathbf{Q}$ (which we shall call the meanings of the logical constants) are taken as, respectively, the mappings $\underline{\exists}, \underline{\mathbb{Q}}, \simeq$ of the polyadic algebra on $Q$, then because this algebra is induced by a polyadic interpretation we may say that the meanings of the logical constants are fixed pragmatically.

In what sense might the objects of the descriptive signs of the formulas of $Q$ be fixed pragmatically? We shall suggest a partial answer to this question for the formulas themselves, on the assumption that the possible objects of the formulas $p \in \mathbf{Q}$ are the images (propositional functions) $\mu p$ in semantic interpretations $\mu$ of the polyadic algebra on $Q$. Thus if $\Pi$ is a polyadic interpretation of $L$, with core $\pi$ and formulas $Q$, then for all $p, q \in \mathbf{Q} ; e \epsilon \mathbf{L}$, and for all semantic interpretations $\mu$ of $\mathbf{Q}$ (i.e., polyadic homomorphisms $\mu$ of $(\mathbf{Q}, \mathbf{I}, \underline{\mathbf{S}}, \underline{\exists})$, with respect to $\mathbf{E}_{\pi}$, onto a model):

If $\Pi(e(p))=\Pi(e(q))$, then $\mu(p)=\mu(q)$.

The antecedent of (5.1) entails that $\mathbf{E}_{\pi}(p, q)$, from which the consequent follows immediately. Relative to $\Pi$, the antecedent of (5.1) may be taken to express the pragmatic synonymy of $p$ and $q$, and the consequent of (5.1) may be taken to express the semantic synonymy of $p$ and $q$. This result, that pragmatic synonymy entails semantic synonymy, in the sense of (5.1), is suggested as a partial explication of Peirce's semiotic principle that the object of a sign is fixed pragmatically, i.e., by the entire general intended interpretant of the sign. The deeper question naturally arises how to associate with formulas of $Q$ those propositional functions which they might be said to signify relative to specific polyadic interpretations. The answer to this question would evidently be of value for the logical foundations of the philosophy of science. 


\section{REFERENCES}

[1] Halmos, P. R., Algebraic Logic, Chelsea Publishing Company, New York (1962).

[2] Quine, W. V., Mathematical Logic, Harvard University Press (1958), pp. 109-115.

[3] Sweet, A. M., "The pragmatics of monadic quantification," Notre Dame Journal of Formal Logic, vol. 10 (1969), pp. 31-46.

Rutgers, The State University

Newark, New Jersey 